Abstract PS2:26 Table 1 Immunoglobulins autoantibodies and pro-inflammatory cytokines in SLE-SS, SLE-noSS and population controls

\begin{tabular}{|c|c|c|c|c|}
\hline & $\begin{array}{c}\text { Controls } \\
\mathrm{N}=322 \\
\text { median (IQR) or } \\
\mathrm{N}(\%)\end{array}$ & $\begin{array}{c}\text { SLE-SS } \\
\mathrm{N}=117 \\
\text { median (cQR) or } \\
\mathrm{N}(\%)\end{array}$ & $\begin{array}{c}\text { SLE-noSS } \\
\text { N=387 } \\
\text { median (IQR) or } \\
\text { N(\%) }\end{array}$ & $\begin{array}{c}\text { p-value } \\
\text { SLE-SS } \\
\text { vs. } \\
\text { SLE-noSS }\end{array}$ \\
\hline IgA total $\mathrm{g} / \mathrm{L}$ & $2.1(1.5 \cdot 2.8)$ & $2.9(1.8-4.3)$ & $2.7(1.9 \cdot 3.6)$ & 0.38 \\
\hline IgG total g/L & $10.9(9.5-12.2)$ & $14.5(10.4-18.3)$ & $12.4(9.8-15.8)$ & 0.009 \\
\hline IgM total g/L & $1.1(0.8-1.6)$ & $1.0(0.5 \cdot 1.6)$ & $0.9(0.6 \cdot 1.5)$ & 0.89 \\
\hline $\begin{array}{l}\text { anti-dsDNA \% positive } \\
(+)\end{array}$ & $5(1.6)$ & $36(31.3)$ & $154(41)$ & 0.06 \\
\hline anti-Ro52 \%+ & $3(0.9)$ & $56(47.9)$ & $84(21.8)$ & $<0.0001$ \\
\hline anti-Ro60 \% + & $5(1.6)$ & 69(59) & $137(35.9)$ & $<0.0001$ \\
\hline anti-La/SSB \% + & $10(3.1)$ & $44(37.6)$ & 69(18) & $<0.0001$ \\
\hline anti-Sm \% + & $I(0.3)$ & $19(16.2)$ & $75(19.5)$ & 0.42 \\
\hline anti-RNP $68 \%+$ & $0(0)$ & $11(9.4)$ & $40(10.4)$ & 0.74 \\
\hline Rf IgG \%+ & $10 / 261(3.8)$ & $17 / 80(21.2)$ & $35 / 259(13.5)$ & 0.09 \\
\hline Rf IgM \% + & 14/283(4.9) & $32 / 83(38.6)$ & $56 / 281(19.9)$ & 0.0005 \\
\hline Rf IgA $\%+$ & $12 / 282(12.4)$ & $34 / 74(45.9)$ & $75 / 267(28.0)$ & 0.004 \\
\hline TNF- $\alpha \mathrm{pg} / \mathrm{mL}$ & $2.3(2.0-2.8)$ & $4.9(3.6-7.1)$ & $4.4(3.0 \cdot 6.0)$ & 0.008 \\
\hline IL-6 pg/mL & $0.5(0.4-0.7)$ & $1.5(0.8 \cdot 3.0)$ & $1.1(0.6 \cdot 2.0)$ & 0.009 \\
\hline MCP-4 pg/mL & $55.8(40.8-80.5)$ & $94.9(66.9 \cdot 131.3)$ & $74.7(52.4-120.0)$ & 0.019 \\
\hline MIP-1 $\beta \mathrm{pg} / \mathrm{mL}$ & $43.7(33.4-56.4)$ & $81.1((54.8-123.6)$ & $68.9(50.3-105.1)$ & 0.021 \\
\hline IL12/IL-23p40 pg/mL & $131.2(99.8-179.5)$ & $211.3(141.4-363.8)$ & $177.1(119.6274 .5)$ & 0.032 \\
\hline IP-10 pg/mL & $351.9(259.2-476.4)$ & $808(536.1911)$ & $726(440-1471)$ & 0.036 \\
\hline
\end{tabular}

\section{PS2:27 ANTIBODIES TO CARBAMYLATED VIMENTIN IN PATIENTS WITH SYSTEMIC LUPUS ERYTHEMATOSUS ARE ASSOCIATED WITH RENAL INVOLVENMENT}

FR Spinelli, T Colasanti, S Truglia, A Pecani, F Ceccarelli, F Miranda, E Moscarelli, R Mancini, C Perricone, C Alessandri, G Valesini, F Conti. Sapienza Università di Roma, Dipartimento di Medicina Interna e Specialità Mediche, Reumatologia, Rome, Italy

10.1136/lupus-2018-abstract.75

Vimentin is a cytoskeletal protein expressed by mesenchymal cells, including endothelial and renal tubular cells. Antibodies to vimentin were described in $10 \%-53 \%$ of patients with Systemic Lupus Erythematosus (SLE). Vimentin has been proposed as a target of the in situ immune response in lupus nephritis. Post-translational modifications increase the immunogenicity of vimentin, as demonstrated by the detection of antimodified-vimentin antibodies in rheumatoid arthritis. Carbamylation is a non-enzymatic post-translational modification (addition of a cyanate group on lysine and arginine residues), which has been linked to NETosis. The role of carbamylated vimentin (Car-Vim) as an antigenic target in SLE has not been evaluated yet.

Aim of the study was to assess the prevalence of anti-CarVIm and to investigate any association with clinical and serological features in SLE patients.

We enrolled SLE diagnosed according to 1997 ACR criteria. Clinical features, autoantibodies profile and disease activity according to SLEDAI $2 \mathrm{~K}$ - were collected. Patients' sera were tested for anti- Car-Vim by a home-made enzyme-linked immunoassay. Data were expressed as mean \pm standard deviation or median (interquartile range) when appropriate. Mann-Whitney and Chi square test were applied to investigate differences in anti- carbamylated vimentin prevalence and serum levels. P value $<0.05$ was considered statistically significant.

We enrolled 109 SLE patients (102F:7M, mean age 39.4 \pm 12.6 years, mean disease duration $10.5 \pm 9.5$ years, mean SLEDAI $2 \mathrm{~K} 5 \pm 5.5$ ). Table 1 summarises the main clinical and serological features. Overall, 30/109 patients (27.5\%) were positive for anti-Car-Vim. The prevalence of anti-Car-Vim was significantly higher in patients with lupus nephritis (18/44) compared to those without $(12 / 66)(41.8 \%$ vs $18.2 \%$, $\mathrm{p}=0.006)$; moreover, anti-Car-Vim serum levels were significantly higher in patients with lupus nephritis [2561 (1783) OD] compared to those without [1970 (1123) OD; $\mathrm{p}=0.0178]$. No difference was found in prevalence or titre of anti-Car-Vim in presence/absence of other clinical or serological manifestations. No correlation between anti-Car-Vim serum levels and SLEDAI $2 \mathrm{~K}$ was found.

Higher prevalence and serum levels of anti-carbamylated vimentin antibodies in patients with lupus nephritis confirm the role of vimentin as a target of the immune response in glomerulonephritis and suggest their possible role as a biomarker of kidney involvement in SLE.

Abstract PS2:27 Table 1 Clinical and serological feature of the patients at the time of enrolment

\begin{tabular}{lc}
\hline Clinica/seroligical feature & $\mathbf{N}(\%)$ \\
\hline Artrhritis & $15(13.8)$ \\
Skin involvement & $16(14.7)$ \\
Lupus nephritis & $43(39.4)$ \\
CNS lupus & $7(6.4)$ \\
Serositis & $3(2.7)$ \\
Hematological disorders & $21(19.3)$ \\
Anti-dsDNA+ & $39 / 74(52.7)$ \\
Low complement levels & $35 / 61(57.4)$ \\
\hline
\end{tabular}

\section{PS2:28 AUTOANTIBODY PROFILING IN PROSTVAC AND IPILIMUMAB TREATED PROSTATE CANCER PATIENTS REVEALS POTENTIAL BIOMARKERS OF IMMUNE- RELATED ADVERSE EVENTS}

${ }^{1} \mathrm{P}$ Budde, ${ }^{2} \mathrm{~J}$ Marte, ${ }^{1} \mathrm{H}-\mathrm{D}$ Zucht, 'S Bhandari, 'M Tuschen, ${ }^{1} \mathrm{P}$ Schulz-Knappe, ${ }^{2} \mathrm{~J}$ Gulley, ${ }^{3} \mathrm{C}$ Heery, ${ }^{2} \mathrm{R}$ Madan, ${ }^{2} \mathrm{~J}$ Schlom. ${ }^{1}$ Protagen AG, Dortmund, Germany, Dortmund, Germany; ${ }^{2}$ National Cancer Institute, National Institutes of Health, Bethesda, MD, USA; ${ }^{3}$ Bavarian Nordic, Inc., Morrisville, NC, USA

\subsection{6/lupus-2018-abstract.76}

Purpose Autoantibodies (AAB) targeting self-antigens can be found in two clinically and immunologically opposing diseases, autoimmune diseases and cancer. While in autoimmune diseases, the immune system is hyperactivated against self-antigens, many tumours suppress the anti-tumour immune response. The therapeutic cancer vaccines PSA-Tricom (Prostvac) is designed to generate an antigen-specific tumour response in metastatic castration-resistant prostate cancer (mCRPC), which is in phase 3 testing. To further augment the immune response, combination therapies of Prostvac with ipilimumab are currently tested in clinical studies. Ipilimumab is an antibody that blocks the immune checkpoint molecule cytotoxic T-lymphocyte-associated antigen 4 (CTLA-4). However, 
treatment with ipilimumab is associated with immune-related adverse events (irAEs). ${ }^{1}$ Since there are no biomarkers for predicting irAEs, we investigated $\mathrm{AAB}$ profiles as biomarkers associated with irAE in mCRPC patients treated with prostvac and ipilumumab combination therapy.

Methods Serum samples from 24 mCRPC patients treated with prostvac and ipiliumab therapy were tested for the presence of serum autoantibodies against 842 preselected antigens. Candidate antigens comprise immune-related and cancer signalling pathway proteins, autoimmune disease antigens, and tumor-associated antigens (TAA). Samples were collected prior to treatment (T0 samples), at 3 and 6 month. IrAEs included rash, elevated aminotransferases, neutropenia, diarrhoea, colitis and endocrine irAEs. Overall survival was also captured and correlated with AABs. Autoantibody levels were measured by Luminex FlexMap3D bead based multiplex protein arrays ${ }^{2}$ and data were analysed by significance analysis of microarrays (SAM), Partial least squares regression (PLS) and Pearson's correlation.

Results In total, 87 AABs were found that were significantly different in patients with irAEs and those without irAEs (SAM $|\mathrm{d}|>2.5$; Pearson's correlation $|3|>0.35)$. PLS analysis revealed that $\mathrm{AABs}$ associated with irAEs were also associated with overall survival. Gene ontology analysis of pathways, molecular function and cellular localization revealed that AABs predicting irAEs target cancer, cell cycle, cell adhesion and apoptotic pathways. We also found elevated levels of AABs in patients who do not experience irAEs. Interestingly, these 40 $\mathrm{AABs}$ target proteins that are involved in inflammatory, adaptive and cellular immune response pathways or are autoimmune disease antigens.

Conclusions $\mathrm{AABs}$ that target antigens involved in cancer signalling pathways are associated with irAEs following prostvac plus checkpoint inhibitor combination therapy. In contrast, AABs targeting immune response pathways were found in patients who do not develop iRAEs and may counteract the action of inflammatory molecules. Similarly, anti-cytokine $\mathrm{AABs}$ have been found in autoimmune diseases, were they appear to counteract the pathological effects of cytokines. ${ }^{3}$ Further studies in larger sample sets are needed to confirm these findings.

\section{REFERENCES}

1. Madan RA, et al. Lancet Oncol 2012;13(5):501-8.

2. Budde $P$, et al. Lupus 2016;25:812-22.

3. Cappellano G, et al. Am J Clin Exp Immunol 2012;1(2):136-46.

\section{PS2:29 THE INCIDENCE AND POSSIBLE RISK FACTORS OF LOW BONE MINERAL DENSITY IN KOREAN PATIENTS WITH SYSTEMIC LUPUS ERYTHEMATOSUS}

C Suh, J Jung, W Baek, S Lee. Ajou Univeristy School of Medicine, Suwon, South Korea

\subsection{6/lupus-2018-abstract.77}

Patients with systemic lupus erythematosus (SLE) are vulnerable to bone loss and fractures, and frequencies of low bone mass reported in $4 \%$ to $74 \%$, variably according to study design and ethnicity. The objective of this study was to evaluate the incidence of low bone mineral density (BMD) and association of clinical factors in Korean patients with SLE. In total, 138 female patients with SLE in 5 hospitals and 165 female healthy controls (HCs) were recruited. All SLE patients fulfilled the 1997 American College of Rheumatology classification criteria for SLE. The osteopenia and osteoporosis was based with the WHO criteria on dual-energy X-ray absorptiometry. The mean age of female SLE patients was 49.7 \pm 11.3 years, and age, weight, and height were not different with those of HCs. Ninety-two (66.7\%) patients with SLE had osteopenia, and $32(23.2 \%)$ patients had osteoporosis, however only $25(15.2 \%)$ HCs had osteopenia $(\mathrm{p}<0.001)$. The SLE patients with osteopenia were older $(53.8 \pm 13.2$ vs $48 \pm 13.7$ years, $\mathrm{p}=0.011)$, lower weight $(54.2 \pm 9.9$ vs 58.3 $\pm 8.4 \mathrm{~kg}, \mathrm{p}=0.008$ ), and took higher cumulative doses of glucocorticoids $\quad(2,332.9 \pm 4,964.2$ vs $\quad 711.4 \pm 2,185.6 \mathrm{mg}$, $\mathrm{p}=0.006)$ than those not. On multivariate regression analysis, age (odds ratio $(\mathrm{OR})=1.039, \mathrm{p}=0.027$ ) and weight $(\mathrm{OR}=0.957, \mathrm{p}=0.038)$ were associated with osteopenia. The incidence of osteopenia was significantly higher in Korean patients with SLE. In addition, age and weight were independent risk factors of osteopenia in SLE.

\section{PS2:30 SYSTEMIC LUPUS ERYTHEMATOSUS PATIENTS WITH POSITIVES AUTOANTIBODIES WITH REMISSION OR LOW ACTIVITY EXHIBIT BOTH LOWER INTERFERON ALPHA AND INTERLEUKIN-10 LEVELS}

${ }^{1} \mathrm{E}$ Grau, ${ }^{2} \mathrm{M}$ Fernandez Matilla, ${ }^{1} \mathrm{FM}$ Ortiz Sanjuan, ${ }^{1} \mathrm{CM}$ Feced Olmos, ${ }^{1} \mathrm{E}$ Labrador Sanchez, ${ }^{2} \mathrm{~N}$ Fernandez-Llanio Cornella, ${ }^{1}$ I Chalmeta Verdejo, ${ }^{1} \mathrm{~K}$ Arevalo Ruales, ${ }^{1} \mathrm{R}$ Negueroles Albuixech, ${ }^{1} \mathrm{~J}$ Ivorra Cortes, ${ }^{1} \mathrm{JJ}$ Fragio Gil, ${ }^{1} \mathrm{I}$ Martinez Cordellat, ${ }^{1} \mathrm{R}$ Gonzalez Mazario, 'L Gonzalez Puig, ${ }^{1} \mathrm{C}$ Alcañiz Escandell, ${ }^{1} \mathrm{C}$ Najera Herranz, ${ }^{1} \mathrm{I}$ Canovas Olmos, ${ }^{1} \mathrm{E}$ Vicens Bernabeu, ${ }^{1} \mathrm{JE}$ Oller Rodriguez, ${ }^{1} \mathrm{M}$ de la Rubia Navarro, ${ }^{2} \mathrm{JA}$ Castellano Cuesta, ${ }^{3} \mathrm{~V}$ Fornes Ferrer, ${ }^{3} \mathrm{D}$ Hervas Marin, ${ }^{1} \mathrm{JA}$ Roman Ivorra. ${ }^{1}$ Rheumatology Department. HUP La $\mathrm{Fe}$, Valencia, Spain; ${ }^{2}$ Rheumatology Section. Hospital Arnau de Vilanova, Valencia, Spain; ${ }^{3}$ Biostatistics Unit. IIS La Fe, Valencia, Spain

\subsection{6/lupus-2018-abstract.78}

Purpose The aim of this study is to assess the clinical and molecular differences in SLE patients with positives autoantibodies and with low clinical activity or in clinical remission compared to the group with clinical activity.

Methods A cross-sectional, observational study of patients diagnosed of SLE according to SLICC 2012 criteria was performed. In these patients a complete blood-test was made, and clinical data by personal interview was collected. We analysed the serum concentration of IL10, BLyS and INF1A by colorimetric methods. Biostatistical analysis was performed with R 3.3.2.

Results We selected 130 SLE patients with serological manifestations (defined by RELESSER study) out of 142 SLE patients. 91 cases showed low activity or remission (SLEDAI <6) and 39 presented moderate or high activity (SLEDAI $>6$ ).

SLE patients with positives autoantibodies without clinical activity showed significantly lower anti-dsDNA levels $(p=0.006)$, lower complement consumption $(p=0.003)$ and lower accumulated damage evaluated by SLICC score $(p=0.041)$. No differences on time of evolution in both groups were observed.

In addition, SLE patients with positives autoantibodies without clinical activity exhibit significantly lower levels of IL10 $(p<0.001)$ and INF1A $(p=0.019)$. No differences on BLyS levels in both groups were observed.

Finally, SLE patients with positives autoantibodies with clinical activity present more mucocutaneous lesions $(p=0.014)$, 